Brazilian Journal of Applied Technology for Agricultural Science, Guarapuava-PR, v.9, n.2, p.71-77, 2016

\section{Cientific Paper}

\section{Resumo}

O eixo cardan é uma das mais eficientes formas de transferir potência do trator para o acionamento de implementos. Para evitar acidentes o mesmo deve ser revestido com um protetor que se compõe de: a) cones em cada uma das extremidades do eixo cardan; b) capa protetora revestindo o tubo telescópico; c) corrente ou dispositivo de retenção equivalente; d) pictogramas ou sinalização de segurança. O objetivo deste trabalho foi verificar as condições de uso desses protetores em propriedades agrícolas do estado de São Paulo. Dos 112 conjuntos trator-implemento analisados, 77 apresentaram algo caracterizado como sendo "proteção" do eixo. Nesta pesquisa, os cones, os tubos, os dispositivos de retenção e a sinalização de segurança foram observados visando identificar a condição (integridade, danificação, ausência) de cada parte. Dos componentes analisados, os cones e os tubos foram os que mais apresentaram danos (63,6 e 71,4\%, respectivamente). Os danos mais comuns nos cones foram trincas, cortes e amassados. Até mesmo amarração com arame ou corda e improvisação com vasilhame plástico foram encontrados. Alguns tubos estavam incompletos, cortados ou soltos sobre o eixo cardan. No caso dos dispositivos de retenção (correntinha) e dos pictogramas, a ausência (50,6 e 53,2 \%, respectivamente) é a situação mais comum. Também foram encontradas improvisações feitas com arames e cordas para o dispositivo de retenção. O baixo percentual da condição "intacto" aponta para a péssima qualidade do material do protetor confirmada por alegações de usuários de que alguns protetores duram menos de seis meses e que isto não os estimula a substituí-los quando danificados.

Palavras chaves: máquina agrícola, segurança de máquinas, proteção de cardan

\section{Assessment of agricultural power take-off (pto) drive shafts guards in field conditions}

Received at: 21/04/16
1 Eng. agrícola, pesquisadora científica do Centro de Engenharia e Automação do Instituto Agronômico (CEA/IAC). Rod. Dom
Gabriel P. B. Couto KM 65 CEP 13212-240-Jundiaí, SP, Brasil. Email: imcorrea@iac.sp.gov.br
2 Eng. agrônomo, Pesquisador científico aposentado do CEA/IAC. Jundiaí, SP, Brasil. Email: cmoreira38@hotmail.com
3 Técnico agrícola do CEA/IAC. Jundiaí, SP, Brasil. Email: sergiorfilipini@yahoo.com.br
4 Eng. agrícola, pesquisador científico do CEA/IAC. Jundiaí, SP, Brasil. Email: rcmello@iac.sp.gov.br
5 Eng. agrônomo, Assistente técnico de pesquisa do CEA/IAC. Jundiaí, SP, Brasil. Email: ppontes@iac.sp.gov.br

Applied Research \& Agrotechnology v9 n2 may/aug. (2016)

Print-ISSN 1983-6325 (On line) e-ISSN 1984-7548 
Corrêa et al. (2016)

the poor quality of the protective material, confirmed by claims of users who say that some guards last less than six months and that it does not encourage them to replace them when damaged.

Key words: agricultural machinery, safety machinery, drive shaft guard

\section{Evaluación de la protección de eje cardan agrícola en campo}

\section{Resumen}

El eje cardan es una de las más eficientes formas de transferir potencia del tractor para el accionamiento de implementos. Para evitar accidentes el mismo debe ser revestido con un protector que se compone de: a) conos a cada una de las extremidades del eje cardan; b) capa protectora revistiendo el tubo telescópico; c) corriente o dispositivo de retención equivalente; d) pictogramas o señalización de seguridad. El objetivo de este trabajo fue verificar las condiciones de uso de esos protectores en propiedades agrícolas del estado de São Paulo. De los 112 conjuntos de tractor-implemento analizados, 77 representan algo característico como siendo "protección" de eje. En esta investigación, los conos, los tubos, los dispositivos de retención y la señalización de seguridad fueron observados buscando identificar la condición (integridad, damnificación, ausencia) de cada parte de los componentes analizados, los conos y los tubos fueron los que más presentaban daños (63,6 y $71,4 \%$, respectivamente). Los daños más comunes en los conos fueron trincas, cortes y aplastados. Hasta mismo amarres con alambre o cuerda y improvisación con envase plástico fueron encontrados. Algunos tubos estaban incompletos, cortados o sueltos sobre el eje cardan. En el caso de dispositivos de retención (correa) y de los pictogramas, la ausencia ( 50,6 y 53,2\%, respectivamente) es la situación más común. También fueron encontradas improvisaciones hechas con alambres y cuerdas para el dispositivo de retención, el bajo porcentaje de condición "intacto" apunta para la pésima calidad del material del protector confirmada por alegaciones de usuarios de que algunos protectores duran menos de seis meses y que esto no los estimula a substituirlos cuando sufren daños.

Palabras Clave: máquina agrícola, seguridad de máquinas, protección de cardan

\section{Introdução}

A transmissão a cardan criada no início do século XX (ATHANASIOV et al., 2006) é uma das mais eficientes formas de transferir potência do trator para o acionamento de máquinas à distância, tais como roçadoras, trilhadoras de cereais, colhedoras de forragens, pulverizadores hidráulicos, entre outros equipamentos. A transmissão compõe-se basicamente de duas juntas universais unidas por elemento de conexão que consiste de dois tubos ou de um tubo e um eixo sólido, introduzidos um no outro, passíveis de movimento telescópico. A transmissão se dá através da chamada "junta universal", ou junta cardânica, composta por dois terminais (também chamados garfos ou luvas) acoplados a cada um dos eixos (do trator e do implemento) unidas por uma cruzeta.

O protetor da transmissão a cardan é composto de cones nas extremidades do eixo, de capa protetora ao longo do tubo telescópico e de dispositivos de retenção além de pictogramas ou sinalização de segurança. CALVO e DEBOLI (2012) citando ANDRONI et al. (1960) dizem que o primeiro sistema de proteção surgiu na década de 60, embora se possa supor que alguma proteção já existia antes disso, pois segundo TINKER et al (1992) já havia legislação na
Grã-Bretanha (HMSO, 1957) estabelecendo requisitos para a proteção da TDP e do eixo cardan, que deveriam entrar em vigor nos anos seguintes.

A possibilidade de transmitir potência (torque e rotação) em ângulo aos implementos agrícolas por meio do eixo cardan ampliou a aplicação do trator na agricultura, porém, ainda é uma das principais fontes de risco e de acidentes no trabalho agrícola, pois o uso do protetor nem sempre corre no campo. Entretanto, quando existente, é comum encontrá-lo com alguma parte quebrada ou trincada fazendo com que perca a sua função.

A utilização inadequada deste equipamento também pode resultar em acidentes graves, sendo que diversos deles estão noticiados na mídia virtual (GUILLEN, 2011; PATRIARCA, 2014, ANON (2016), CARLOS (2016) entre outros) e na literatura técnica (BEER et al., 2007; CORRÊA et al., 2003, DEBIASI e SCHLOSSER, 2002). Contribuem para isso o fato do acoplamento/desacoplamento serem feitos manualmente e a falta de uso do protetor do eixo cardan. No primeiro caso, cita-se a operação manual de conexão e desconexão trator x TDP, que é realizada em espaço reduzido entre o trator e o implemento, o que expõe o operador a condições de fadiga e desconforto (POCHI e FEDRIZZI, 2008). Neste caso, os autores dizem que o operador deve levantar e 


\section{Avaliação da proteção de eixos... Assessment of agricultural power take-off... Evaluación de la protección de eje...}

colocar corretamente no lugar a junta respectiva e empurrá-la até que o travamento ocorra e que o desenvolvimento de dispositivos automáticos pode reduzir esta intervenção manual.

No segundo caso, a ausência do protetor do eixo cardan pode provocar acidentes ainda mais graves quando este está em funcionamento. Se a roupa ou cabelo se enrolam no eixo girando a pessoa é puxada imediatamente por ele. O corpo é girado em torno do eixo podendo ocorrer amputação de braços e pernas, escalpelamento, lesões medulares, outras injúrias ou mesmo a morte.

Na avaliação de THOMAS e BUCKMASTER (2003) os maiores fatores de causa deste tipo de acidente, ocorrem em operação estacionária devido à ausência ou danos no protetor, podendo, também, envolver pessoas próximas à operação, com consequiências severas: morte (3\%), amputação (26\%) e fraturas (32\%).

A legislação brasileira através das normas regulamentadoras NR 31 (BRASIL, 2011) e NR 12 (BRASIL, 2012) impõe que "o eixo cardan deve possuir proteção adequada, devendo estar em perfeito estado de conservação em toda a sua extensão, fixada na tomada de força da máquina (ou do trator) desde a cruzeta até o acoplamento do implemento ou equipamento".

Embora as operações agrícolas devam ser realizadas por pessoas adultas, a participação de menores de idade os expõe aos mesmos riscos, como mostra trabalho de BEER et al. (2007). Os autores analisaram dados da Universidade de Purdue (EUA) com registros de acidentes relacionados à transmissão a cardan ocorridos de 1970 a 2004 buscando identificar aqueles que envolviam pessoas com idade abaixo de 18 anos. Neste estudo, 22,0 \% dos casos com idade conhecida foram identificados nesta faixa de idade. Foram relatados casos de amputação de membros (quase 50\% de ocorrência), lesões na espinha e fratura de ossos em acidentes envolvendo eixo sem-fim em elevadores e transportadores de grãos.

Na Inglaterra, 201 máquinas acionadas pela tomada de potência (TDP) foram inspecionadas por TINKER et al. (1992) sendo achado danos em 78\% das proteções. CECCHINI et al. (2011) demonstraram em pesquisa na região central da Itália, a situação dos protetores de cardan em uso, onde somente os mais novos, de até dois anos, encontravamse em condições adequadas, enquanto que os demais encontravam se em condições precárias, apresentando dispositivos de retenção ausentes em $72 \%$ dos casos; - pictogramas legíveis em apenas
39\% deles e nos demais, sinalização de segurança danificada ou perdida.

Não se tem conhecimento de levantamento semelhante no Brasil. Assim, o objetivo deste trabalho foi verificar as condições de uso de protetores de eixo cardan em propriedades agrícolas do Estado de São Paulo.

\section{Material e método}

Para analisar as condições dos protetores de cardan, buscou-se caracterizar tais equipamentos em diversas propriedades agrícolas do Estado de São Paulo. Foram amostrados 112 exemplares de eixos cardans em nove municípios de São Paulo (Jundiaí, Campinas, Mogi das Cruzes, Mogi Mirim, Botucatu, Capão Bonito, Aguaí, Matão e Jarinu). A escolha dos municípios foi aleatória levando em conta a distância e aproveitando a conveniência de outras ações nos locais. As informações foram coletadas no final de 2012 por meio de formulário especialmente desenvolvido para tal.

Para cada um dos protetores encontrados buscou-se fazer uma caracterização da marca/modelo e ano de fabricação bem como a presença e condições dos dispositivos de proteção e outras observações. Foram analisados os seguintes componentes do protetor: cones de proteção, proteção do tubo telescópico, dispositivo de retenção e os pictogramas ou sinalização de segurança.

Os resultados sobre a presença dos dispositivos de proteção foram expressos em porcentagem do total de protetores encontrados e apresentados em forma gráfica.

\section{Resultados e discussão}

Dos 112 conjuntos trator-implemento analisados, 77 apresentaram algum componente caracterizado como sendo "proteção" do eixo cardan. Os principais implementos que faziam uso de acionamento por transmissão a cardan foram: pulverizadores $(48,21 \%)$, roçadoras $(15,18 \%)$, distribuidores de calcário e esterco $(10,71 \%)$. As demais máquinas, entre elas, semeadoras, adubadoras, tanques de água, trituradores de restos vegetais e outras correspondiam a $13,39 \%$ dos conjuntos analisados.

Uma identificação completa de marca/ modelo e ano de fabricação não foi possível. Dos protetores encontrados 33,77\% não apresentavam 
identificação de marca. Dentre os possíveis de identificar, a distribuição de marcas foi a seguinte: Aemco (32,47\%), MCP-Gicatti (10,39\%), Albarus $(7,79 \%)$, Bondioli e Pavosi (6,49\%), Walterscheid $(6,49 \%)$ e outros dois $(2,60 \%)$ em relação ao total dos protetores. Quanto ao ano de fabricação, em menos da metade $(38,96 \%$, correspondente a 30 exemplares), foi encontrada a informação. Destes trinta exemplares, dezenove $(63,33 \%)$ tinham até dois anos e seis exemplares $(20,00 \%)$ tinham até cinco anos de uso. Comparado à amostra analisada os dezenove exemplares correspondem a $24,67 \%$ do total.

Neste trabalho, o cone, o tubo, o dispositivo de retenção e a sinalização de segurança foram observados visando identificar a condição (integridade, danificação, ausência) de cada parte. As figuras1e 2 sintetizam estes resultados.

Dos componentes analisados, os cones e os tubos foram os componentes que mais apresentaram danos (63,6 e 71,4\%, respectivamente). Os danos mais comuns nos cones são trincas, cortes e amassamentos. Até mesmo amarração com arame ou corda e improvisação com vasilhame plástico (frasco de agrotóxico) foram encontrados para cobrir a caixa de transmissão da roçadora. Alguns tubos estavam incompletos, cortados, soltos sobre o eixo cardan. TINKER et al. (1992) identificaram como causas mais comuns de danos nos tubos, o contato deles com a estrutura das máquinas, como a barra de tração ou com os braços inferiores do sistema de engate de três pontos. No caso dos cones as causas apontadas por TINKER et al. (1992), - como atrito na proteção da TDP ou na proteção do eixo no lado do implemento, ou ainda por terem sido cortados para permitir a lubrificação das juntas universais - foram situações também encontradas no presente levantamento.

O baixo percentual da condição "intacto" em

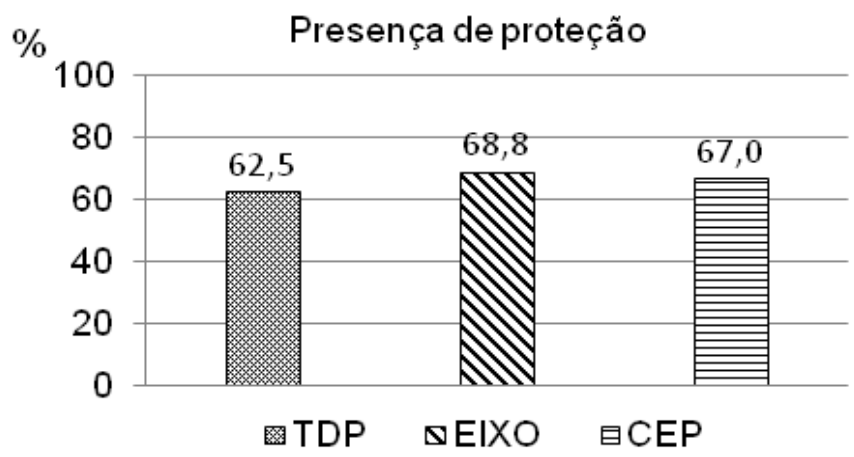

Figura 1. Presença de proteção em 112 eixos cardan, incluindo a tomada de potência do trator (TDP), o tubo do eixo cardan e a conexão de entrada de potência no implemento (CEP).

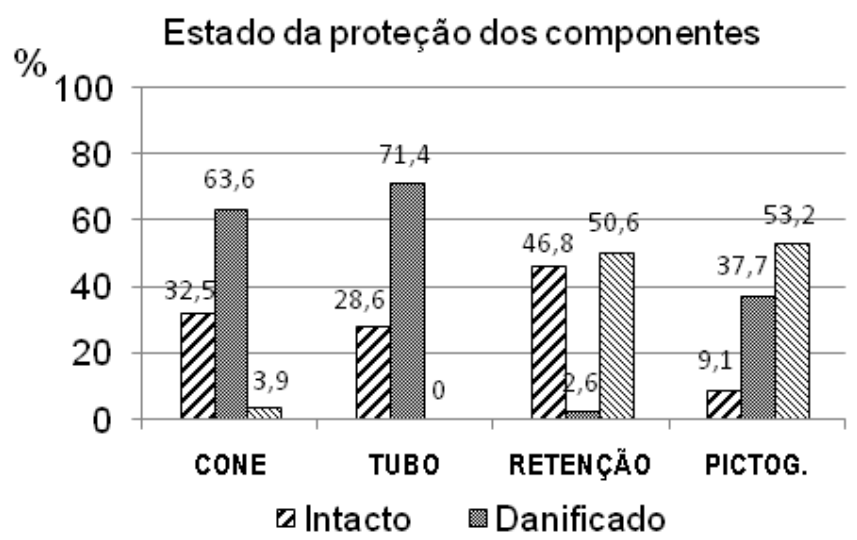

Figura 2. Estado dos componentes dos protetores de eixo cardan agrícola. 


\section{Avaliação da proteção de eixos... \\ Assessment of agricultural power take-off... Evaluación de la protección de eje...}

cones e tubos aponta para a péssima qualidade do material do protetor confirmada por alegações de usuários de que alguns protetores duram menos de seis meses e que isto não os estimula a substituílos quando danificados. A não substituição dos protetores quando danificados também é mencionada por MONTEIRO (2011) e ATHANASIOV et al. (2006).

No caso dos dispositivos de retenção (correntinha) e dos pictogramas, a ausência (50,6 e $53,2 \%$, respectivamente) é a situação mais comum. Também foram encontradas improvisações feitas com arames ou cordas como substitutos do dispositivo de retenção. Exemplos como estes, chamados "gambiarras" são mencionados por MONTEIRO (2011) para operação com a tomada de potência. No levantamento feito por TINKER et al. (1992), apenas $40 \%$ das correntes de retenção ainda estavam no lugar.

Devido ao baixo número de protetores com identificação da idade de uso, não foi possível estabelecer uma relação quali/quantitativa entre a idade e o estado do protetor, como foi feito por CHECHINI et al. (2011).

O problema da qualidade do material é antigo. HYLAND-MCAGUIRE (1994) ao analisar alguns casos de acidentes envolvendo a tomada de potência de tratores, faz algumas sugestões para reduzir o risco de acidente. Dentre elas estão, a necessidade de treinamento dos agricultores, o uso de roupas adequadas e melhorias no projeto do protetor da tomada de potência que a façam mais difícil de ser removida e menos propensa à deterioração do material com a idade.

Para melhorar a qualidade deste componente no Brasil ele deveria passar pela realização de ensaios de resistência e durabilidade. Entretanto, a ausência de laboratórios especializados, que possam prestar auxílio aos fabricantes interessados, dificulta ação neste sentido. Particularmente, devido à incidência da radiação solar ser abundante no território brasileiro, atenção especial deveria ser dada ao material plástico utilizado na confecção do protetor de modo a torná-lo mais resistente aos raios ultravioleta dando-lhe talvez maior resistência mecânica.

\section{Outras observações}

Somando-se à baixa qualidade do protetor de eixo cardan, observou-se que também os usuários não mantêm o equipamento em boas condições de armazenagem quando em não uso. Dependendo do local onde o eixo se apóia quando em descanso, pode ocorrer danificação dos pictogramas ou amassamento dos cones. Em muitos casos, os eixos cardan, com proteção ou sem proteção, são deixados em contato com o solo, sujando-se de terra.

Há também inúmeras queixas sobre a dificuldade de acoplamento, na montagem e desmontagem bem como na lubrificação das cruzetas fazendo com que alguns usuários aumentem o furo de engraxe dos cones para acessar as cruzetas.

\section{Conclusão}

Dos componentes analisados, os cones e os tubos foram os componentes que mais apresentaram danos (63,6 e 71,4\%, respectivamente). Os danos mais comuns nos cones são trincas, cortes e amassamentos. Até mesmo amarração com arame e improvisação com vasilhame plástico foram encontrados. Alguns tubos estavam incompletos, cortados, soltos, girando junto sobre o eixo cardan.

No caso dos dispositivos de retenção (correntinha) e dos pictogramas, a ausência (50,6 e $53,2 \%$, respectivamente) é a situação mais comum. Também foram encontradas improvisações feitas com arames e cordas para o dispositivo de retenção. O baixo percentual da condição "intacto" aponta para a péssima qualidade do material do protetor confirmada por alegações de usuários de que alguns protetores duram menos de seis meses o que não os estimula a substituí-los quando danificados.

\section{Referências}

ANON. Criança de 11 anos morre em acidente de trator. PORTAL TRI. Dionísio Cerqueira, SC. 30.jul.2015. Disponível em < http:/ / www.portaltri.com.br/1/noticias/4/geral/44868/crianca-de-11-anos-morre-em-acidente-comtrator> Acesso em 05 de julho 2016.

ATHANASIOV, A., FRAGAR, L., GUPTA, M. Farm machinery injury: Power take-off shaft guards. Rural Industries Research and Development Corporation and the Australian Centre for Agricultural Health and Safety. Report. Farm Publication n. 06/035. Sydnei, AU, ISBN 1741512980 ISSN 1440-6845 Sep. 2006, 15p. 
BRASIL. MINISTÉRIO DO TRABALHO E EMPREGO, 2011. Portaria MTE $\mathrm{n}^{\circ}$ 2.546, de 14 de dezembro de 2011. Disponível em:<htt:http://portal.mte.gov.br/data/files/8A7C812D33EF459 C0134561C307E1E94/ NR-31\%20\%28atualizada\%202011\%29.pdf>. Acesso em: 02 de agosto de 2012.

BRASIL. MINISTÉRIO DO TRABALHO E EMPREGO, 2010. Portaria SIT n. . 197, de 17 de dezembro de 2010. Disponível em:<http:/ / portal.mte.gov.br/data/files/8A7C812D350A C6F801357BCD39D2456A/NR-12\%20 \%28atualizada\%202011\%29\%20II.pdf Acesso em 02 de agosto de 2012.

BEER, S.R.; DEBOY, G.R. FIELD, W.E. Analysis of 151 Agricultural Driveline-Related Incidents Resulting in Fatal and Non-Fatal Injuries to U.S. Children and Adolescents Under Age 18 from 1970 through 2004. Abstract. 2007. Disponível em<http:/ / www.ncbi.nlm.nih.gov/ pubmed/17555204>. Acesso em: 15 de agosto de 2012

CALVO, A., DEBOLI, R. The sunrise of agricultural ergonomics and safety studies in Italy and in . Europe. In: International Conference about Safety Health and Welfare in Agriculture and in Agro-food Systems (RAGUSA SHWA 2012), Sep. 3-6, 2012, Ragusa - Italy. Proceedings. Anais, p.25-33. 2012

CARLOS, L. Jovem tem pé amputado em acidente com trator. RADAR ALTO VALE. Rio do Sul, SC. 04.abr.2016. Disponível em:http:/ / www.radaraltovale.com/noticia/antenado/jovem-tem-o-pe-amputado-em-acidentecom-trator-26094\#.V4POTPkrLIU . Acesso em 05 de julho de 2016.

CECCHINI, M; MONARCA, D.; BIONDI, P.; COLANTI, A.; BRENCIAGLIA, A. Safety of Tractor PTO Drive Shafts: Survey on maintenance on a Sample of Farms in Central Italy. In: XXXIV Commission Internationale de 1'Organisation Scientifique Du Travail em Agriculture (CIOSTA), V Conference (CIGR). 29 jun-01 jul, 2011. Viena, Austria. Paper. 6p. Disponível em: https://www.researchgate.net/publication/277124996_Efficient_ and_safe_production_processes_in_sustainable_agriculture_and_forestry_XXXIV_CIOSTA_CIGR_V_ Conference_2011_Safety_of_Tractor_PTO_Drive_Shafts_Survey_on_Maintenance_on_a_Sample_of_Farms_ in_Central Acesso em: 11 de janeiro de 2012

CORRÊA, I. M, YAMASHITA, R. Y., RAMOS, H. H., FRANCO, A. V. F. Perfil dos acidentes rurais em agências do INSS de São Paulo no ano 2000. Revista Brasileira de Saúde Ocupacional, São Paulo, SP, v. 28, n.107/108, p. 39-57, 2003. http://dx.doi.org/10.1590/S0303-76572003000200005

DEBIASI, H. SCHLOSSER, J. F. Acidentes com tratores. Cultivar Máquinas, Pelotas, RS, n. 12, p. 28-33, maio/ junho de 2002.

GUILLEN, F. Acidente com trator deixa agricultor em estado grave em Mamborê. Gazeta do Povo. Curitiba, 05.ago, 2011. Disponİvel em< http://www.gazetadopovo.com.br/vida-e-cidadania/maringa/acidentecom-trator-deixa-agricultor-em-estado-grave-em-mambore-c0usq02un6lqqeum7vuswk18u> Acesso em 05 de julho de 2016.

HER MAJEST'S STATIONARY OFFICE - HMSO. The agriculture (Power take-off) regulations. Statutory Instrument $\mathrm{N}^{\mathrm{o}}$ 1386. HMSO, London, 1957. 4p. ISBN 0111003733

HYLAND-MCAGUIRE, P. Farm accidents involving power take-off devices. Journal of Accident and Emergency Medicine. Elsevier, San Diego, CA, n.11, p. 121-124, 1994. DOI:10.1136, Online ISSN1472-0213

MONTEIRO, L. de A. Acidentes com tratores agrícolas: Parte II. Jornal Dia de Campo, 04 fev. 2011. Disponível em <http://www.diadecampo.com.br/zpublisher/materias/Materia.asp?id $=23623 \&$ secao $=$ Colunas $\% 20$ e\%20Artigos>. Acesso em 06 de julho de 2016.

PATRIARCA, P. Trabalhador rural morre em acidente com trator. 18 set.2014. JCNet-Jornal da Cidade de Bauru. Disponível em <http:/ / www.jcnet.com.br/Regional/2014/09/trabalhador-rural-morre-em-acidentecom-trator.html> Acesso em 06 de julho de 2016

POCCHI, D.; FEDRIZZI, M. A device for the automation of the engagement between trator's Power -take-off and PTO-drive shaft. In: International Conference about Innovation Technology to Empower Safety, Health and Welfare in Agriculture and Agro-food Systems, Sep., 15-17, 2008. Ragusa, Itália. Proceedings. 7p. 2008

THOMAS, R. S.; BUCKMASTER, D. R. Relative Safety of Traditional Agricultural Tractor Power Take-Off (PTO) Drivelines Compared to Fluid Power - A Review. Journal of Agricultural Safety and Health, 9 (3): 241-250. ASABE, St. Joseph, Mich.: Aug. 2003. DOI: 0.13031/2013.13689 


\section{Avaliação da proteção de eixos... Assessment of agricultural power take-off... Evaluación de la protección de eje...}

TINKER, D. B., DWYER, M. J., SANDERS, M. P. Causes of damage to Power take-off shaft guards - Alternative Power supply tractor to implement. Silsoe, Bedford. HSE Contract Report n ${ }^{\circ}$ 43/1992. 89p. 